\title{
El aprendizaje-servicio en la práctica docente de la Geografía: propuestas para la gestión turística del Parque Natural Chera-Sot de Chera (Valencia)
}

\author{
Sergio Bellés Monserrat ${ }^{a}$, Jaime Escribano Pizarro ${ }^{b}$ y Néstor Vercher Savall ${ }^{c}$ \\ a'Universitat de València bemonser@alumni.uv.es \\ bInstituto Interuniversitario de Desarrollo Local - Dpto. Geografía. Universitat de València. jaime.escribano@uv.es \\ ${ }^{\mathrm{c}}$ Instituto Interuniversitario de Desarrollo Local - Dpto. Geografía. Universitat de València. nestor.vercher@uv.es
}

\section{HXP HQ}

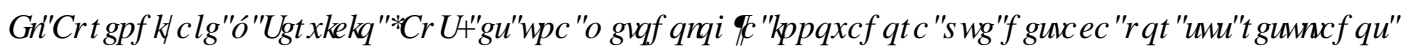

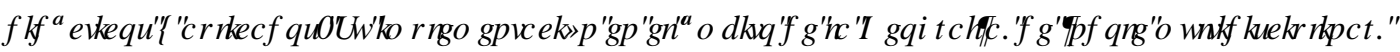

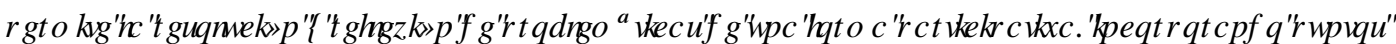

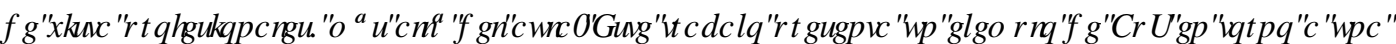

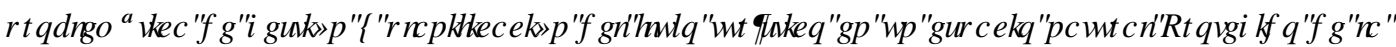

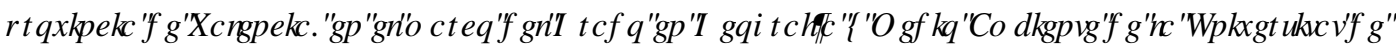

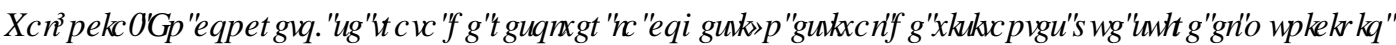

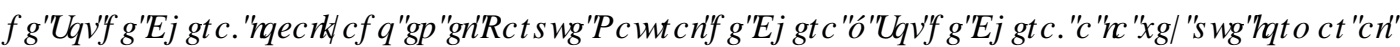

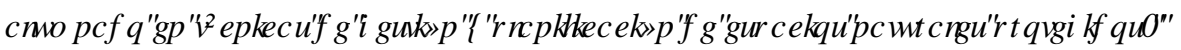

\section{DOEWVFOYH}

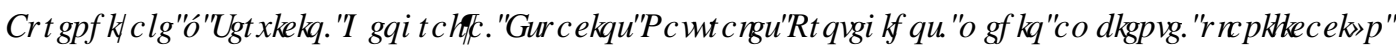
UसUTRUDOI

\section{\$EWWFW}

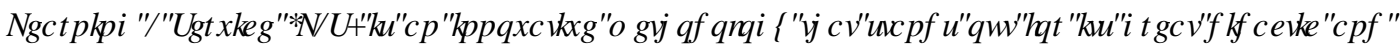

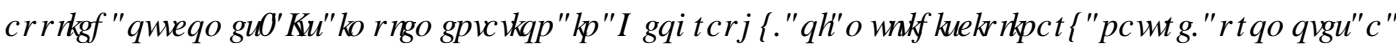

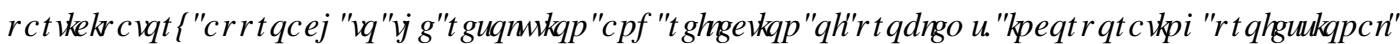

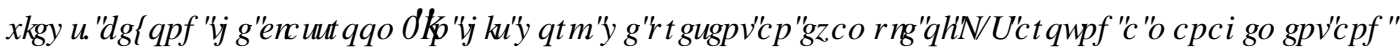

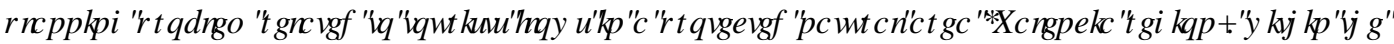

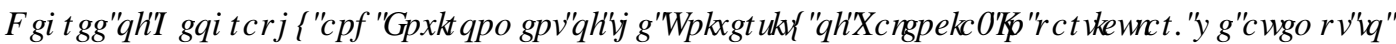

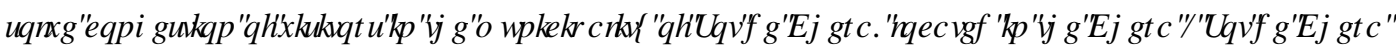

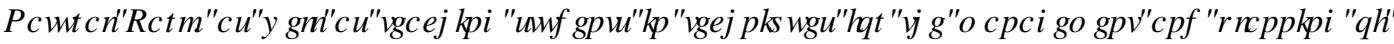
SLRUFUFEQDUKLODUHVD

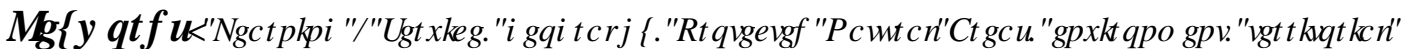
SQDQRQJW 


\section{Introducción}

El Aprendizaje - Servicio (ApS) es una metodología de trabajo que se caracteriza por su elevado nivel de aplicabilidad. Se trata de una propuesta pedagógica basada en la solidaridad, en la participación ciudadana activa y que, si es planificada adecuadamente, puede ser una manera novedosa de aprender contenidos, adquirir competencias y modificar actitudes (Benavides y Quintana, 2002; Dorsey, 2007). Se trata de una

enseñanza que no solo promueve la formación ciudadana y el aprendizaje de valores, sino que, además, insta a una forma de aprender que da sentido y relevancia social al conocimiento (Ministerio de Educación de la Nación, 2012). Así, se puede afirmar que es una metodología didáctica que combina la educación con el ofrecimiento de un servicio a la comunidad, involucrándose a los participantes en temas reales y de verdadera necesidad del entorno (Lara y Delgado, 2016; Mahon et al., 2020).

En el caso que nos atañe, el ApS cobra más importancia si cabe debido a que, por una parte, se trata de estudios de índole geográfica cuya visión es multidisciplinar y transversal y a que, por otra parte, se trata de aprendizajes realizados durante la etapa universitaria (García y Martínez, 2014).

Las tareas descritas se enmarcan en la asignatura optativa de Espacios Naturales Protegidos (código 35006), de seis créditos ECTS, que forma parte de la titulación de Grado en Geografía y Medio Ambiente de la Universitat de València (España). Esta materia fue cursada por 20 estudiantes durante el segundo cuatrimestre del curso académico 2018-2019. En su contexto se trata de una metodología innovadora, pues no se había implementado hasta la fecha en este grado universitario. Si bien, existen propuestas recientes en el mismo grado que también apuestan por la introducción de métodos docentes innovadores (Zornoza y Vercher, 2021).

En el marco de la búsqueda de la mejora continua de la docencia, son numerosas las universidades que se comprometen con la práctica del ApS y promueven la creación de unidades desde las cuales asesorar e impulsar la puesta en marcha de iniciativas que insten a docentes e investigadores a trabajarlo (Martínez et al., 2018). Este es el caso del presente proyecto, respaldado desde el Vicerrectorado de Ocupación y Programas Formativos de la Universitat de València ${ }^{1}$.

Las tareas abordadas se basaron en la gestión del flujo turístico estival del municipio de Sot de Chera (Valencia). Este forma parte del Parque Natural y Geológico de Chera - Sot de Chera y, durante los fines de semana, festividades y época estival, sufre graves problemas de congestión turística como resultado de la llegada de un gran número de visitantes a sus zonas de baño que acaban ocasionando unas graves afecciones ambientales y sociales. Esta problemática, debido al escaso presupuesto municipal, aún no se había abordado pese a que trasciende de una administración y afecta a la vida cotidiana de este municipio rural. Además, es un asunto que, de tratarse de manera adecuada, puede: i) favorecer la descongestión turística, maximizando el disfrute de visitantes con la mínima molestia para la población local; ii) reducir su impacto ambiental, muy necesario puesto que es un espacio que cuenta con gran valor ecológico y patrimonial; y contribuir a la dinamización económica local, muy necesaria tanto en este como en otros municipios rurales de características similares.

\footnotetext{
${ }^{1}$ El aprendizaje-servicio en la práctica geográfica: aproximación al Uso Público del Parque Natural Chera-Sot de Chera (Valencia) (UV-SFPIE RMD18-954293). Proyecto de Innovación Educativa y Calidad Docente del Servei de Formació Permanent i Innovació Educativa, de la Universidad de Valencia (Vicerrectorado de Políticas de Formación y Calidad Educativa). Tipo de proyecto: renovación de metodologías docentes. Duración: 14/02/2019 - 30/09/2019 (7 meses). IP: Jaime Escribano (UV). Equipo: Néstor Vercher (UV), José Vicente Sánchez (UV), Paula Poveda Beneyto (GVA) y Pablo Arjona Pérez (UV). Dotación: 400,00€.
} 
De este modo, las tareas llevadas a cabo para la consecución satisfactoria de este ApS se focalizaron en conocer todas las plazas de aparcamiento disponibles cercanas a zonas de baño, las plazas de aparcamiento requeridas por la población local y las restantes que quedan disponibles para los visitantes. También se estimó la capacidad de carga física de las zonas de baño pudiéndose dar un número aproximado de visitantes que pueden acceder al municipio sin causar graves daños en el ecosistema medioambiental.

\section{Objetivos}

El método de ApS que aquí se aplica combina el trabajo en el aula con el de campo. En estas salidas, pese a que no exista vinculación con el espacio, destaca el interés que suele crear en los estudiantes; se puede observar cómo, cuanto mayor es el nivel de estudios alcanzado, mayor será la escala territorial que suscite interés (García Hernández, 2020).

Atendiendo a la problemática planteada y teniendo en cuenta el bagaje académico del alumnado, los objetivos que se persiguen con la propuesta aquí expuesta de ApS son los siguientes:

- Demostrar que los contenidos teórico-conceptuales adquiridos durante el Grado en Geografía y Medio Ambiente permiten adquirir una base sólida para trabajar en el ámbito de la planificación territorial y medioambiental. Se persigue que los/las estudiantes aprendan a poner en práctica los conocimientos adquiridos durante los estudios universitarios, realizándose una primera aproximación con el mundo laboral y de transferencia de conocimiento. Es decir, se trata de fomentar la aplicación de conocimientos teórico - prácticos con atención a las demandas de la sociedad y de las administraciones públicas.

- Atender a una necesidad manifestada por el Equipo Técnico encargado de la gestión del Parque Natural y Geológico de Chera-Sot de Chera la cual está estrechamente vinculada con los estudios del Grado en Geografía y Medio Ambiente. Fruto del contacto previo entre los docentes y los técnicos del Parque, se comunicó la problemática que supone la intensa y reiterada llegada de visitantes al municipio durante las principales festividades y los meses de verano. Dicha problemática, en mayor o menor grado habitual en todos los Parques Naturales próximos a grandes ciudades, como es el caso, debe ser abordada en un estudio de Uso Público de los recursos del mismo, haciendo hincapié en sus posibilidades de aprovechamiento sostenible. Sin embargo, este es un documento apenas desarrollado en el parque en cuestión, puesto que carece de los recursos y medios necesarios. Por este motivo, se traslada la posibilidad de colaborar en la actualización y desarrollo de este estudio.

- Fomentar la participación de los/las estudiantes de la asignatura de Espacios Naturales Protegidos en la resolución de problemas del territorio valenciano desde la óptica de la gestión y planificación de un área protegida. Con la realización de estas tareas se permite que los/las estudiantes puedan ayudar y aprender a resolver problemáticas de espacios de gran valor ecológico de su entorno territorial más próximo, cuyas repercusiones son multidisciplinares, yendo más allá de las puramente ambientales. Esta situación favorece la toma de conciencia territorial y motivación del alumnado, lo que puede derivar en unos aprendizajes y resultados más satisfactorios.

Los objetivos planteados se alinean de manera directa con el concepto del ApS: aprender contenidos, adquirir competencias y habilidades, y modificar actitudes. 


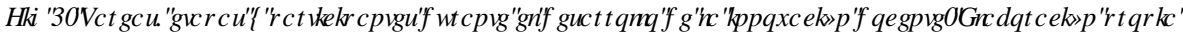

\begin{tabular}{|c|c|c|c|c|c|c|c|c|}
\hline $\begin{array}{c}\text { Segunda } \\
\text { quincena de } \\
\text { enero }\end{array}$ & $\begin{array}{c}\text { Primera } \\
\text { quincena de } \\
\text { febrero }\end{array}$ & $\begin{array}{c}\text { El } 18 \text { y } 22 \\
\text { febrero de } \\
2019\end{array}$ & $\begin{array}{c}\text { Entre el } 25 \\
\text { febrero y el } \\
14 \text { marzo de } \\
2019\end{array}$ & $\begin{array}{c}\text { Entre el } 25 \\
\text { y el } 29 \\
\text { marzo de } \\
2019\end{array}$ & $\begin{array}{c}\text { Viernes } 5 \\
\text { de abril de } \\
2019\end{array}$ & $\begin{array}{c}\text { Entre el } 8 \\
\text { y el } 12 \text { de } \\
\text { abril de } \\
2019\end{array}$ & $\begin{array}{c}\text { Entre el } 15 \\
\text { abril y el } 3 \\
\text { mayo de } \\
2019\end{array}$ & $\begin{array}{c}6 \text { de } \\
\text { mayo de } \\
2019\end{array}$ \\
\hline $\begin{array}{l}\text { TAREAS } \\
\text { - Reunión con las } \\
\text { autoridades y } \\
\text { técnicos } \\
\text { municipales y } \\
\text { del Parque } \\
\text { Natural de Chera } \\
\text { - Sot de Chera } \\
\text { para concretar } \\
\text { aspectos } \\
\text { prácticos a } \\
\text { desarrollar en la } \\
\text { posterior visita } \\
\text { PARTICIPANTES } \\
\text { - Docentes, } \\
\text { alcalde del } \\
\text { municipio, } \\
\text { técnicos } \\
\text { municipales y } \\
\text { del Parque } \\
\text { Natural }\end{array}$ & $\begin{array}{l}\text { TAREAS } \\
\text { - Realización de } \\
\text { dos sesiones en } \\
\text { el aula para } \\
\text { definir las líneas } \\
\text { de trabajo y la } \\
\text { propuesta de } \\
\text { organización de } \\
\text { los equipos de } \\
\text { trabajo de } \\
\text { alumnos } \\
\text { PARTICIPANTES } \\
\text { - Docentes y } \\
\text { estudiantes de la } \\
\text { asignatura }\end{array}$ & \begin{tabular}{|l|} 
TAREAS \\
- Reunión general \\
con los alumnos \\
para explicar en \\
detalle el \\
proyecto y \\
discutir con ellos \\
aspectos de su \\
desarrollo. \\
Además, se \\
aprovechará para \\
concretar los \\
equipos de \\
trabajo \\
conformados por \\
los estudiantes \\
PARTICIPANTES \\
- Docentes y \\
estudiantes de la \\
asignatura
\end{tabular} & $\begin{array}{l}\text { TAREAS } \\
\text { - Breves sesiones } \\
\text { de preparación en } \\
\text { torno a las fichas } \\
\text { de trabajo que } \\
\text { servirán para la } \\
\text { recogida de } \\
\text { información in } \\
\text { situ; serán } \\
\text { explicadas y } \\
\text { discutidas con el } \\
\text { alumnado. Estas } \\
\text { sesiones de } \\
\text { trabajo integrarán } \\
\text { nociones básicas } \\
\text { sobre el territorio } \\
\text { de estudio y cómo } \\
\text { se desarrollará la } \\
\text { actividad } \\
\\
\text { PARTICIPANTES } \\
\text { - Docentes y } \\
\text { estudiantes de la } \\
\text { asignatura }\end{array}$ & $\begin{array}{l}\text { TAREAS } \\
\text { - Los docentes se } \\
\text { reúnen para } \\
\text { concretar los } \\
\text { últimos aspectos } \\
\text { de la salida, y } \\
\text { verificar que } \\
\text { todos los grupos } \\
\text { de alumnos han } \\
\text { adquirido los } \\
\text { conocimientos } \\
\text { teóricos y } \\
\text { prácticos } \\
\text { necesarios } \\
\text { previos para el } \\
\text { desarrollo de la } \\
\text { jornada } \\
\text { PARTICIPANTES } \\
\text { - Docentes de la } \\
\text { asignatura }\end{array}$ & $\begin{array}{l}\text { TAREAS } \\
\text { - Jornada de } \\
\text { trabajo de campo } \\
\text { en Sot de Chera. } \\
\text { Acompañamiento } \\
\text { y apoyo continuo } \\
\text { de la técnica del } \\
\text { Parque Natural } \\
\text { PARTICIPANTES } \\
\text { - Docentes, } \\
\text { estudiantes de la } \\
\text { asignatura y } \\
\text { técnica del } \\
\text { Parque Natural }\end{array}$ & $\begin{array}{l}\text { TAREAS } \\
\text { - Sesión de } \\
\text { discusión y } \\
\text { organización de } \\
\text { toda la } \\
\text { información } \\
\text { recabada durante } \\
\text { la salida. Así, en } \\
\text { cada clase, los } \\
\text { docentes podrán } \\
\text { marcar las } \\
\text { directrices para } \\
\text { que los equipos } \\
\text { puedan llevar a } \\
\text { cabo el trabajo } \\
\text { autónomo } \\
\text { adecuadamente } \\
\text { PARTICIPANTES } \\
\text { - Docentes y } \\
\text { estudiantes de la } \\
\text { asignatura }\end{array}$ & $\begin{array}{l}\text { TAREAS } \\
\text { - Trabajo } \\
\text { autónomo y } \\
\text { grupal, } \\
\text { supervisado con } \\
\text { tutorías por los } \\
\text { docentes } \\
\text { PARTICIPANTES } \\
\text { - Docentes y } \\
\text { estudiantes de la } \\
\text { asignatura }\end{array}$ & $\begin{array}{l}\text { TAREAS } \\
\text { - Realización de una } \\
\text { jornada en la que los } \\
\text { diferentes grupos de } \\
\text { trabajo de alumnos } \\
\text { exponen sus } \\
\text { resultados a los } \\
\text { docentes, los técnicos } \\
\text { municipales, la } \\
\text { técnico del Parque } \\
\text { Natural, el director } \\
\text { del Parque Natural y } \\
\text { el alcalde. Asimismo, } \\
\text { se emplea esta jornada } \\
\text { para compartir } \\
\text { valoraciones sobre la } \\
\text { experiencia con los } \\
\text { alumnos participantes } \\
\\
\text { PARTICIPANTES } \\
\text { - Docentes y } \\
\text { estudiantes de la } \\
\text { asignatura, técnicos } \\
\text { municipales y del } \\
\text { Parque Natural y el } \\
\text { Director del Parque } \\
\text { Natural }\end{array}$ \\
\hline
\end{tabular}




\section{Desarrollo de la innovación}

En el marco del método del ApS y los objetivos descritos antes, el recurso de las salidas de campo deviene imprescindible para conocer IQUW la problemática territorial a tratar (Dorsey, 2007; Lara y Delgado, 2016; Rees et al., 2021). A través de las salidas de campo el estudiantado tiene la posibilidad de: i) recoger información directa y real en el ámbito territorial del Parque Natural y Geológico de Chera-Sot de Chera, ii) conocer las necesidades clave de la población local, y iii) aplicar y profundizar en los conocimientos de la asignatura involucrada en el proyecto.

En concreto, se permite integrar los contenidos teóricos con la información recabada durante la visita. Con ello, el alumnado es consciente que se trata de un servicio territorial en favor de un espacio protegido, y que surge de una necesidad local insatisfecha. También se permiten identificar nuevos nichos de empleo y de servicio a la comunidad, e incluso mejorar las técnicas empleadas para atender dichas problemáticas.

De los trabajos de gabinete realizados, se identifican como fundamentales dos tareas: delimitar las áreas de baño y definir las zonas de parking viables. A partir de las medidas de los espacios que se usan para el baño (ver Figura 2) y de considerar de tres escenarios de ocupación para cada usuario de $5 \mathrm{~m}^{2}, 7,5 \mathrm{~m}^{2}$ y $10 \mathrm{~m}^{2}$, aplicando unos cálculos concretos, se obtienen tres capacidades de carga física distintas que presentan una mayor o menor densidad de bañistas. Con el análisis del entorno y la aplicación de distintas normativas de usos del suelo y de riesgos naturales, se pudieron conocer los espacios susceptibles de acoger plazas de aparcamiento.

A partir de estas dos tareas, y con la ayuda de Sistemas de Información Geográfica, se pudieron determinar el número y la localización de las plazas de aparcamiento que son viables realizar en el municipio; estos espacios se pueden ver representados en la Figura 3.

El plan de trabajo y desarrollo de la innovación sigue un orden cronológico y lineal que, JLRWR $P R G R$, comprende la documentación previa del caso (trabajo de gabinete), la salida de campo para conocer IQUW la problemática, las tareas de subsanación de errores y/o modificaciones a realizar después de la visita y, por último, la redacción del informe final, realizado de manera colaborativa por todos los estudiantes mediante medios digitales, y su exposición al equipo técnico del parque. La Figura 1 muestra el detalle de las tareas y actores involucrados en cada etapa del desarrollo de la innovación docente.

Una vez finalizados los trabajos y realizada la exposición a los responsables municipales y del Parque Natural, el profesorado de la asignatura realizó una encuesta para conocer el grado de satisfacción de los alumnos respecto a las tareas realizadas y aquellos aspecto que, en el caso que se decidiese volver a aplicar esta metodología, se pueden mejorar.

El cuestionario se realizó completamente online, utilizando la plataforma Google Forms, y formó parte del proceso de autoevaluación al que los profesores sometieron a la asignatura. Este procedimiento estaba formado por un total de 29 preguntas de las que 15 trataban directamente sobre el ApS; las cuestiones planteadas se podían responder mediante escalas lineales, cuyos indicadores estaban preestablecidos, respuestas cortas o valores numéricos. La acogida entre el alumnado del procedimiento de autoevaluación fue muy buena, puesto que respondió todo el alumnado (20 personas). En la Tabla 1 se indican cuáles fueron las preguntas, la tipología de respuesta que tuvo cada una de ellas y los valores de respuesta. 


\begin{tabular}{|c|c|c|}
\hline Texto de la pregunta & \multicolumn{1}{c}{ Tipología de } \\
respuesta & $\begin{array}{r}\text { Total de } \\
\text { respuestas } \\
\text { (N=20) }\end{array}$ \\
\hline $\begin{array}{c}\text { ¿Crees que el proyecto te ha servido para entender } \\
\text { mejor las problemáticas y conflictos asociados a } \\
\text { Espacios Naturales Protegidos? }\end{array}$ & $\begin{array}{c}\text { Escala lineal (Mucho, } \\
\text { bastante, poco, nada) }\end{array}$ & 20 \\
\hline
\end{tabular}

Espacios Naturales Protegidos?

¿Consideras que has adquirido, asimilado y puesto en práctica habilidades necesarias para desarrollar un estudio aplicado en Espacios Naturales Protegidos?

¿Qué destacarías en la experiencia del proyecto en relación a aprendizajes, habilidades y capacidades desarrolladas y/o puestas en práctica?

Valora el trabajo con tus compañeros/as durante el proyecto en relación a los siguientes aspectos

[Capacidad de resolución de problemas]

Valora el trabajo con tus compañeros/as durante el proyecto en relación a los siguientes aspectos

[Responsabilidad en la entrega de tareas]

Valora el trabajo con tus compañeros/as durante el proyecto en relación a los siguientes aspectos

[Actitud colaborativa y comunicativa]

Valora el trabajo con tus compañeros/as durante el proyecto en relación a los siguientes aspectos [Creatividad e innovación]

¿Estás satisfecho con los resultados del proyecto (el informe)?
Escala lineal (Mucho, bastante, poco, nada) bueno, bueno, regular, malo)

Escala lineal (Muy bueno, bueno, regular, malo)

Escala lineal (Muy bueno, bueno, regular, malo)

Escala lineal (Muy bueno, bueno, regular, malo)

Escala lineal (Mucho, 
Solo aquellos que asistieron a la presentación: ¿Estás satisfecho con los resultados del proyecto (la presentación)?

¿Qué es lo que más te ha gustado/interesado del proyecto?

¿Qué te ha costado más o has encontrado más complejo en el proyecto?

¿Qué aspectos del proceso colectivo de elaboración del proyecto mejorarías?

Considero que este tipo de proyectos aprendizajeservicio deberían aplicarse en otras asignaturas del grado

Considerando lo que has aprendido y tu participación e implicación en el proyecto, ¿qué nota te pondrías?

Valora la experiencia del proyecto en su conjunto
Escala lineal (Mucho, bastante, poco, nada)

Respuesta corta

Respuesta corta

Respuesta corta

Escala lineal (muy de acuerdo, de acuerdo, en desacuerdo)

Valor numérico de 1 a

10

Valor numérico de 1 a

*Solamente 6 alumnos realizaron la exposición del trabajo realizado a las autoridades municipales y del Parque Natural.

En la sección 4. $5 H X W D V$ de este documento se representan aquellas preguntas cuya información resulta ser de mayor utilidad para conocer la satisfacción del alumnado.

\section{Resultados}

El ApS puede ser abordado desde distintas ópticas e implicar actores de muy diversa índole (Mahon et al. 2020). En el caso de Sot de Chera, las partes implicadas fueron el alumnado y los docentes de la materia que vertebra el proyecto, y las autoridades locales y ambientales del entorno del Parque Natural. En concreto, ha sido el alumnado matriculado en la asignatura y las autoridades municipales quienes se han visto más directamente implicados.

Por una parte, los primeros han tenido la oportunidad de realizar un aprendizaje práctico sobre una de las problemáticas más recurrentes que atañen a los espacios protegidos de la Comunidad Valenciana, al mismo tiempo que han conocido una de las diversas posibilidades que ofrece el mercado laboral para los egresados del Grado en Geografía y Medio Ambiente, un factor muy a tener en cuenta ya que se trata de una asignatura de último curso.

Por otra parte, los segundos, tras haber indicado una problemática que les preocupaba, han conseguido obtener un estudio técnico que les sirve de base para poder abordarla, supervisado por profesionales de la geografía, que ha contado con la colaboración de un amplio abanico de agentes locales implicados. El 


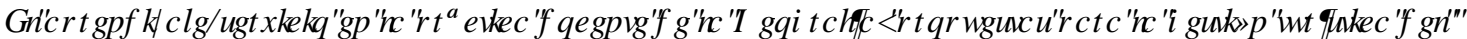 3 DUXH1 DUWO\&KHD6RWCH\&KHDШ9DOPFWD}

प

informe, el cual fue presentado de manera presencial a los responsables municipales y ambientales, incluye las zonas de aparcamiento que son viables (ver Figura 3) atendiendo a criterios ambientales y normativos, para la cual resulta fundamental la propuesta de reordenación del tráfico municipal. Además, también se realiza un estudio sobre la opinión de la población local que pueda ayudar en la toma de decisiones.

\subsubsection{Delimitación}

Para llevar a cabo el estudio de la capacidad de acogida fisica en éstas zonas de baño, el procedimiento seguido ha sido el siguiente. Primeramente, se delimitó la extensión de cada una de las zonas de en las cuales se quiere permitir el baño. La suma total es de $2.449,84 \mathrm{~m} 2$. Éste área se divide en:

- La zona cimentada del río, que a su vez se puede dividir en una zona norte, contigua al pueblo, (Zona 1: Piscinas norte) ocupa un espacio de $1.103,46 \mathrm{~m} 2$, mientras que, en su zona sur, localizada en la otra orilla del río, ocupa un total de $322,15 \mathrm{~m} 2$. (Zona 2: Piscinas sur).

- La parte baja del río, cercana al albergue de El Cerrao y dónde existe un área recreativa, ocupa una extensión de $822,78 \mathrm{~m} 2$. (Zona 3: Área recreativa).

- La zona de baño más alejada del municipio, ubicada en el Paraje de Las Toscas, ocupa un total de 201,45 m2. En esta cabe destacar que, la extensión susceptible de ser ocupada es bastante menor ya que, y a diferencia de las áreas anteriores, existe una abundante vegetación de ribera que reduce la extensión susceptible de ser ocupada por los bañistas.

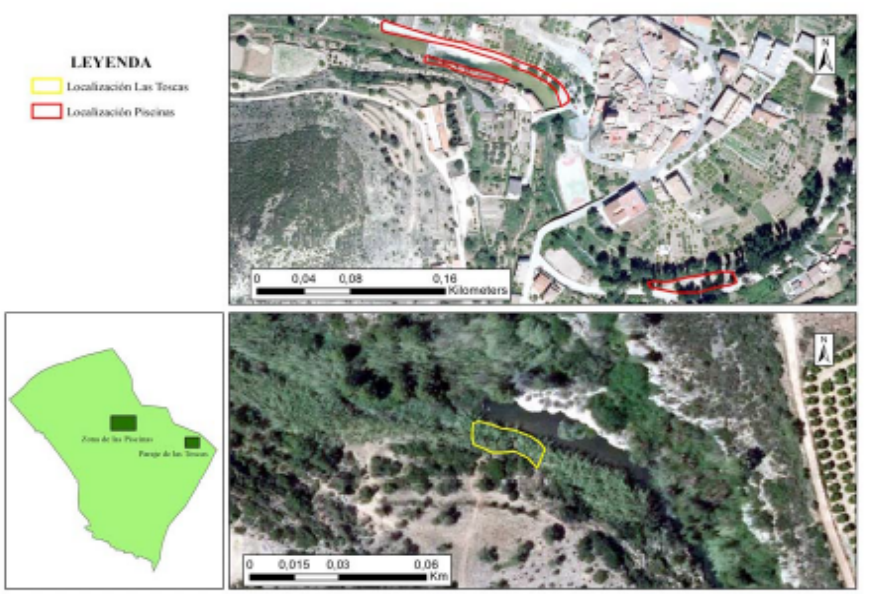

Figura 2. Detalle de localización de las zonas de baño en Sot de Chera. Fuente: elaboración propia.

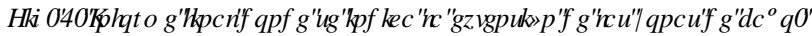




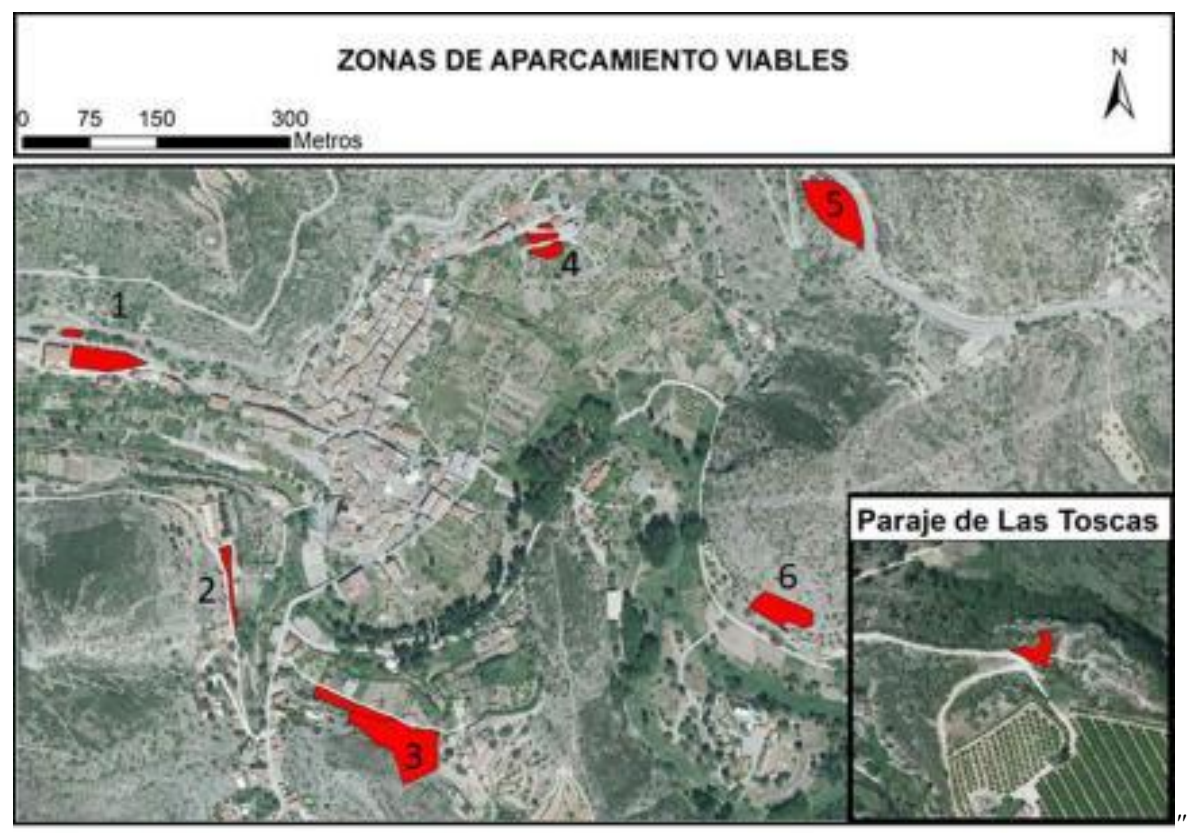

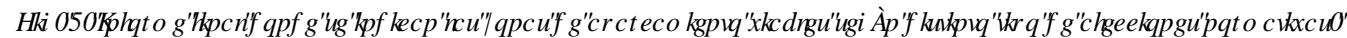

Una vez implementado el ApS, se realizó una encuesta a los estudiantes para conocer su opinión al respecto sobre el trabajo realizado (tanto en cuanto a contenidos y objetivos, como en relación al modo y desarrollo seguido). En líneas generales se puede afirmar que, además de tener unos resultados que responden a los objetivos planteados, la valoración del alumnado ha sido muy positiva. En primer lugar, los conocimientos adquiridos durante el Grado en Geografía y Medio Ambiente, en general, y en la asignatura de Espacios Naturales Protegidos, en particular, permiten contar una base sólida para trabajar en el ámbito de la planificación territorial y medioambiental. Esto se refleja en las Figuras 4 y 5, en las que los/las estudiantes afirman que, gracias a este proyecto ApS, han podido asimilar y poner en práctica las habilidades necesarias para desarrollar un estudio aplicado y real, y han podido entender mejor las problemáticas a las cuales se enfrentan estos territorios de gran valor medioambiental.

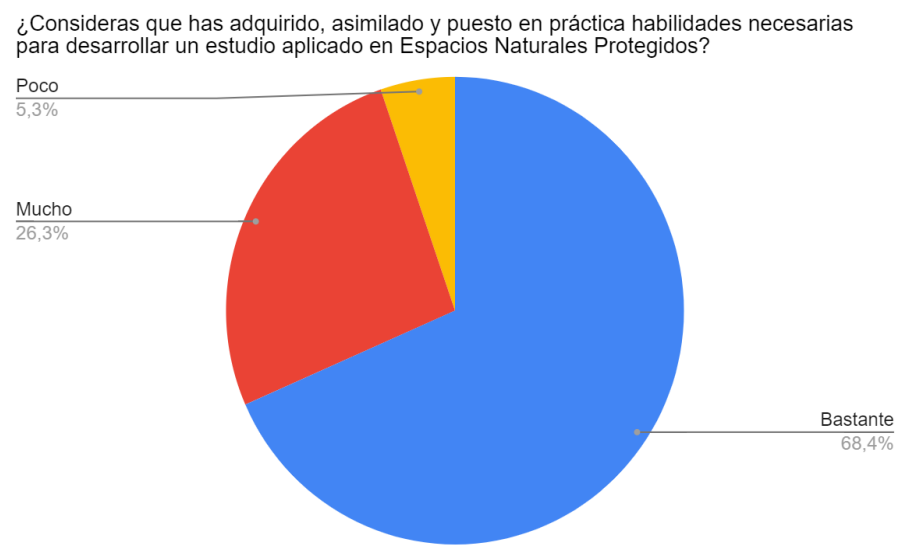

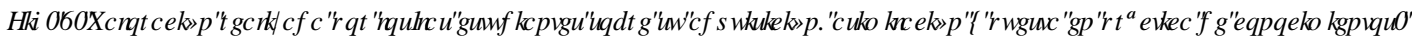




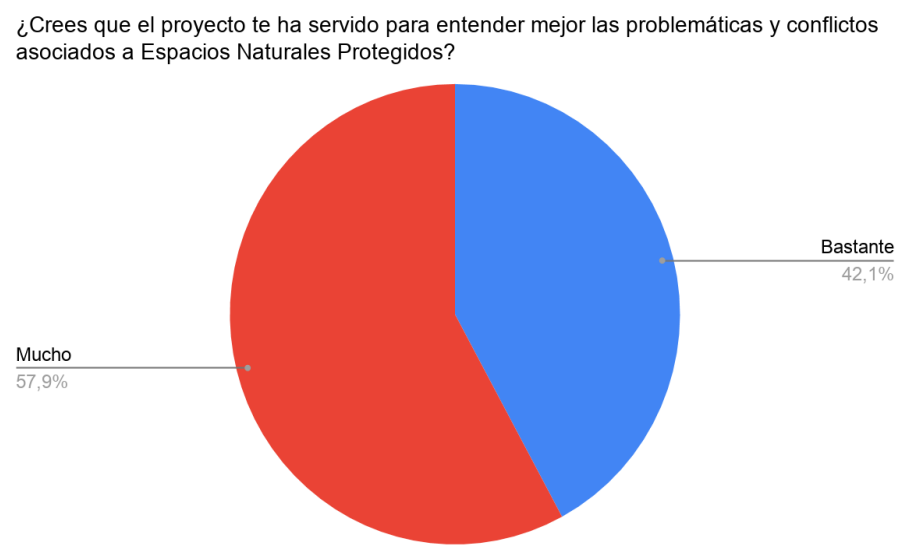

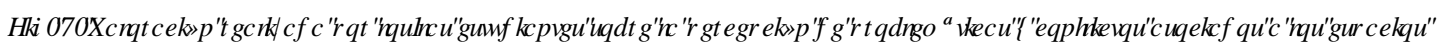
QDUWDOALSLRUIIRRI

En segundo lugar, se puede afirmar que, al mismo tiempo que se ha atendido una necesidad indicada por la sociedad del municipio de Sot de Chera y por los técnicos del Parque Natural, se ha realizado un trabajo académico que, como se puede observar en la Figura 6, ha contado con una valoración satisfactoria por parte del alumnado.

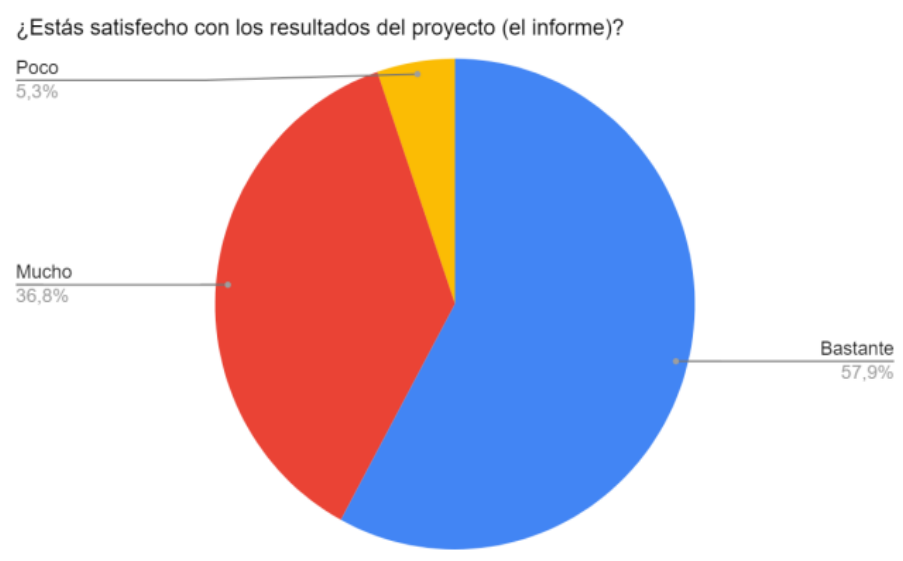

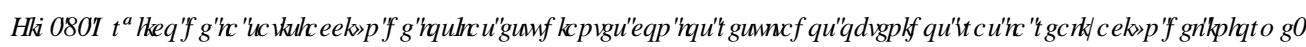

En tercer y último lugar, se ha conseguido fomentar la participación del estudiantado en el desarrollo de la asignatura. Fruto de esto fue la creación de un KDWDJ en la red social Twitter durante la visita al municipio de Sot de Chera, en el que los estudiantes pudieron compartir sus vivencias y aprendizajes durante la jornada en la que se desarrolló la salida de campo. De este modo, se favoreció la participación estudiantil adaptada a las nuevas tecnologías. Además, esta participación también refleja una mejora de la cooperación entre los/las estudiantes. La Figura 7 muestra que la mayor parte del alumnado ha considerado que el trabajo realizado por sus compañeros y compañeras ha sido bueno o muy bueno, reflejando así la buena coordinación y cooperación que se ha formado alrededor de este proyecto. 
Adicionalmente, se debe resaltar que este proyecto ha sido valorado con una nota de 8,5 sobre 10 puntos por los y las estudiantes, y que, todos ellos, consideraron de manera unánime, que esta metodología se debería aplicar en otras asignaturas de este grado universitario (Figura 8).

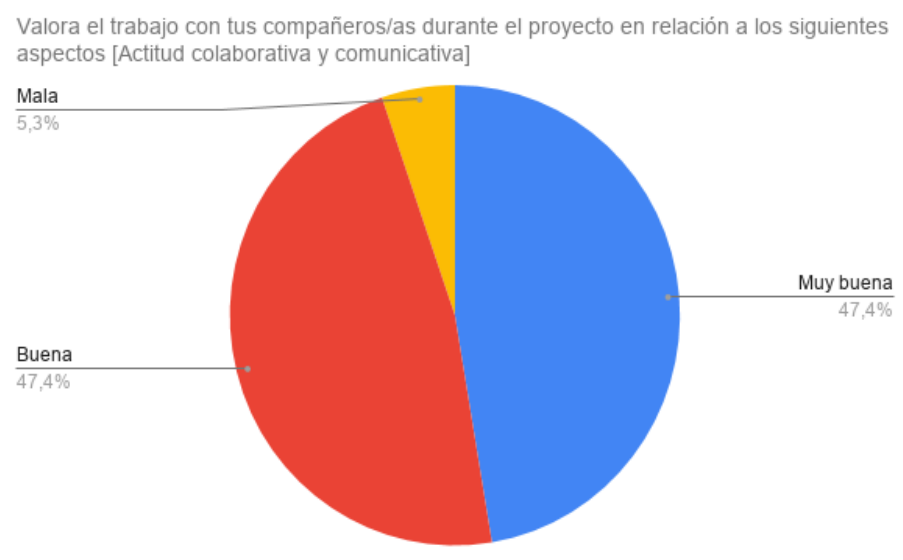

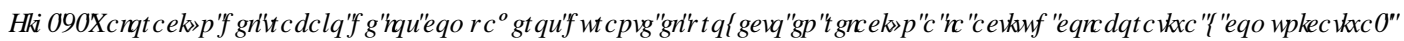

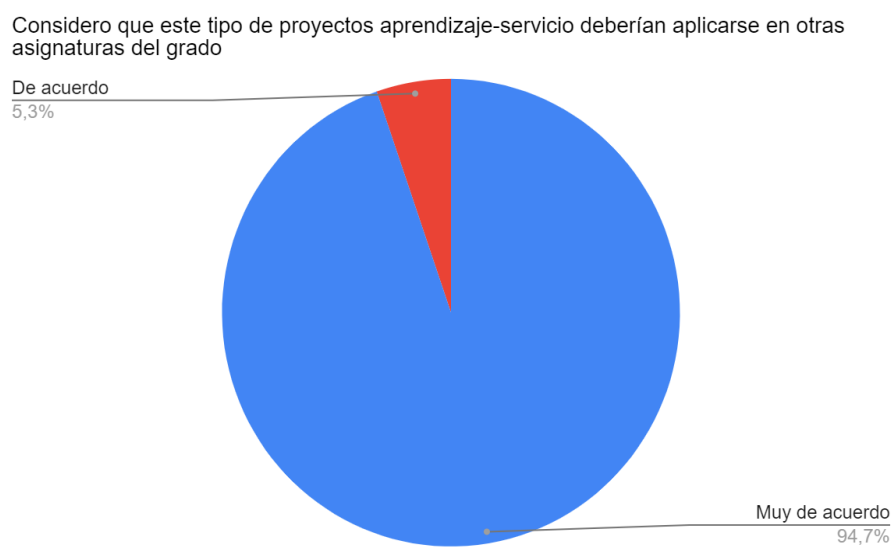

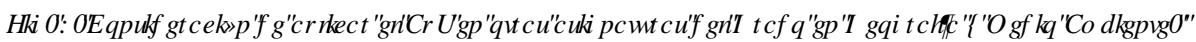

\section{Conclusiones}

Con la realización de este proyecto se ha avanzado en la resolución de la congestión turística del municipio valenciano de Sot de Chera, mediante la aplicación de la metodología de ApS. Como se ha apuntado anteriormente, esta es una metodología didáctica que combina la educación con el ofrecimiento de un servicio a la comunidad y que involucra a los participantes en temas reales y de verdadera necesidad del entorno (Lara y Delgado, 2016; Mahon et al. 2020).

Se ha conseguido que los estudiantes puedan concebir como aplicables aquellos conocimientos adquiridos durante su formación universitaria, resolver una problemática ambiental, económica y social que, de no haber sido por esta propuesta, no se habría resuelto (Dorsey, 2007), y favorecer la participación de los estudiantes en la resolución de problemáticas de espacios naturales protegidos (Martínez y García, 2015). 


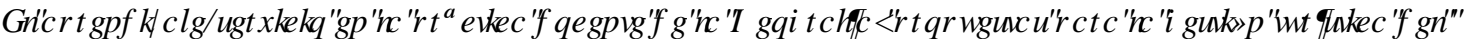 3 DUXH1 DUWO\&KHD6RWCH\&KHDШ9DOPFWD}

[

La realización de estas tareas ha tenido un resultado muy satisfactorio tanto desde el punto de vista de las autoridades municipales y ambientales, como, especialmente, desde el punto de vista de los/las estudiantes.

El buen resultado del ApS en el caso particular expuesto, y a tenor de la demanda reflejada en la Figura 8, el equipo docente de la asignatura ha tratado de dar continuidad y expansión a esta metodología docente. Por ejemplo, durante el curso 2019-2020 se mantuvo el mismo método (ApS) en la misma asignatura, lógicamente con la implicación del equipo técnico del mismo Parque Natural, si bien para una temática distinta: la identificación y catalogación de los recursos fontanales del espacio protegido, lo que habla de la buena acogida que tuvo el diseño y ejecución de la anterior propuesta en la zona ${ }^{2}$. Además, durante el presente curso académico 2020-2021, el ApS se ha expandido a un número mayor de asignaturas, grados y másteres (Desarrollo Local Sostenible, optativa de cuarto curso del Grado en Geografía y Medio Ambiente; Gobierno y Gestión para el Desarrollo Local, optativa de cuarto curso del Grado en Ciencias Políticas y de la Administración Pública; Mercados Locales, Promoción del Empleo y Territorio, asignatura obligatoria del Máster Título Propio en Gestión de Políticas para el Desarrollo del Territorio; y Cooperativas agrarias y desarrollo rural, optativa del Máster en Economía Social (Cooperativas y Entidades No Lucrativas), tratando de atender las necesidades territoriales de emprendimiento y empleabilidad de espacios rurales como Sot de Chera, Tuéjar, Banyeres de la Mariola, etc. ${ }^{3}$

\section{Referencias}

BENAVIDES-VELASCO, C. y QUINTANA-GARCÍA, C. (2002). "Regiones en aprendizaje: ¿Una nueva dimensión territorial de la innovación?" en \%RQHQHFRQ P IFR $]$ GH, \& ( पL, QRLP DFIy Q\&RP HFIDOC ISD RQ, 2722, 19-30.

DORSEY, B. (2007). "Linking Theories of Service-Learning and Undergraduate Geography Education" -RXLQDO RI * HJUSKK, Vol. 100 (3), pp. 124-132. <https://doi.org/10.1080/00221340108978428> [Consulta: 25 de marzo de 2021].

GARCÍA, J. S. (2020). "El Aprendizaje-Servicio en la ciudad: un itinerario didáctico para trabajar las

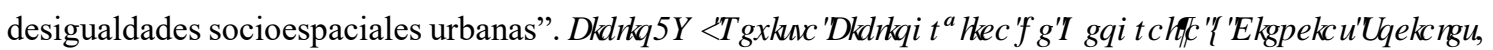
Vol. 25 (1286), 1-26. <https://doi.org/10.1344/b3w.25.2020.29255> [Consulta: 25 de marzo de 2021].

GARCÍA, A. J. y MARTÍNEZ, L. C. (2014). "El aprendizaje-servicio como propuesta didáctica para la propuesta didáctica para la enseñanza de la geografía. Una experiencia de puesta en valor del patrimonio

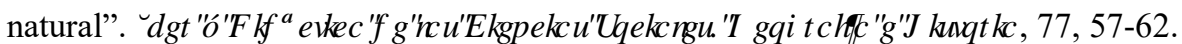

LARA, M. F. y DELGADO, J. J. (2016). “Aplicación del aprendizaje-servicio en el ámbito de la geografía:

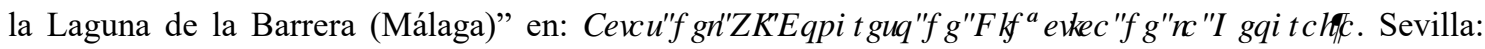
Universidad de Pablo Olavide y Grupo de Didáctica de la Geografía de la AGE. Disponible en $<$ http://hdl.handle.net/10630/12445> [Consulta el 24 de marzo de 2021].

\footnotetext{
${ }^{2}$ Este proyecto se implementó sin apoyo alguno por parte de la Universitat de València o entidad colaborada implicada en el mismo, pese a la procedencia de los participantes (todos estudiantes y profesores de dicha universidad).

3 El desarrollo local en ámbitos rurales: experiencias y buenas prácticas para la empleabilidad y el emprendimiento (UVSFPIE_PID20-1355876). Proyecto de Innovación Educativa y $\quad$ Calidad Docente del Servei de Formació Permanent i Innovació Educativa, de la Universidad de Valencia (Vicerrectorado de Políticas de Formación y Calidad Educativa). Tipo de proyecto: Proyecto de Innovación Docente (PID). Duración: 31/10/2020 - 31/07/2021 (9 meses). IP: Jaime Escribano. Equipo: Javier Esparcia, Néstor Vercher, José Javier Serrano, y Cristina Herraiz. Dotación: 990,00€.
} 
MAHON, M., CONWAY, T., FARELL, M. y MCDONAGH, J. (2020). "Service Learnig As A Means To Develop Geography Graduates Professional Identity”. \$LKH-W Vol. 12 (1), 1-20

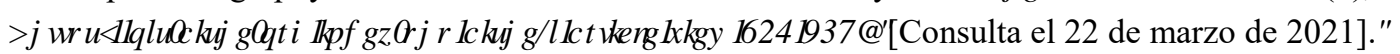

MARTÍNEZ, V., MELERO, N., IBAÑEZ, E. y SANCHEZ, M. C. (2018). ( O\$SUHQ]DDNH6HUIFIRIHQ(D)

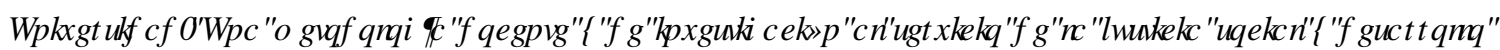
VRWHECH Salamanca: Comunicación Social. < https://rio.upo.es/xmlui/handle/10433/6323> [Consulta el 24 de marzo de 2021].

MARTÍNEZ, L. C. y GARCÍA, A. J. (2015). "Service-learning, educational innovation and geography: an experience in assessing territorial heritage in the Biosphere Reserve of the area known as Ancares Leoneses (Cantabrian mountain range, Spain)". 5HLLDGH) RLP DFly QH, QQRYDFly Q( GXFDWDD8 QIYHUWUD, Vol. 8 (11), 23-30. <http://uvadoc.uva.es/handle/10324/22823> [Consulta el 22 de marzo de 2021].

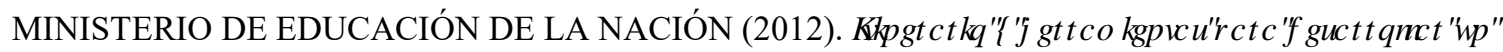

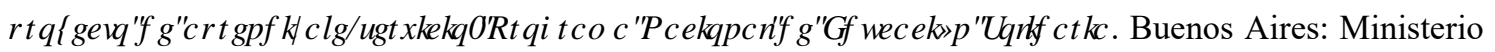
de Educación de la Nación. < http://www.bnm.me.gov.ar/giga1/documentos/EL005050.pdf $>$ [Consulta el 23 de marzo de 2021].

REES, A., HAWTHORNE, T., SCOTT, D., SOLIS, P. y SPEARS, E. (2021). "Toward a Community Geography Pedagody: A Focus on Reciprocal Relationships and Reflection”. - RXLQDORI* HRJWSK $\square 120$, 36-47. <https://doi.org/10.1080/00221341.2020.1841820> [Consulta: 25 de marzo de 2021].

ZORNOZA, C. y VERCHER, N. (2020). "Evaluación de competencias genéricas vinculadas al proceso de elaboración del TFG. Propuesta de rúbrica para el Grado de Geografía y Medio Ambiente de la Universitat de València". \&XDGHQRV* HRJU IIFRV, 60(1), 119-138. <https://doi.org/10.30827/cuadgeo.v60i1.13790>. 